\title{
Phonological coding in word reading: Evidence from hearing and deaf readers
}

\author{
VICKI L. HANSON \\ Haskins Laboratories, New Haven, Connecticut \\ and \\ CAROL A. FOWLER \\ Dartmouth College, Hanover, New Hampshire \\ and Haskins Laboratories, New Haven, Connecticut
}

\begin{abstract}
The ability of prelingually, profoundly deaf readers to access phonological information during reading was investigated in three experiments. The experiments employed a task, developed by Meyer, Schvaneveldt, and Ruddy (1974), in which lexical decision response times (RTs) to orthographically similar rhyming (e.g., WAVE-SAVE) and nonrhyming (e.g., HAVE-CAVE) word pairs were compared with RTs to orthographically and phonologically dissimilar control word pairs. The subjects of the study were deaf college students and hearing college students. In Experiments 1 and 2 , in which the nonwords were pronounceable, the deaf subjects, like the hearing subjects, were facilitated in their RTs to rhyming pairs, but not to nonrhyming pairs. In Experiment 3 , in which the nonwords were unpronounceable, both deaf and hearing subjects were facilitated in their RTs to both rhyming and nonrhyming pairs, with the facilitation being significantly greater for the rhyming pairs. These results indicate that access to phonological information is possible despite prelingual and profound hearing impairment. As such, they run counter to claims that deaf individuals are limited to the use of visual strategies in reading. Given the impoverished auditory experience of such readers, these results suggest that the use of phonological information need not be tied to the auditory modality.
\end{abstract}

There is evidence that, under some experimental conditions, skilled readers with normal hearing access phonological information about the words they read. One such set of experimental conditions was described by Meyer, Schvaneveldt, and Ruddy (1974). In their procedure, subjects are shown pairs of letter strings to which they respond "yes" if both letter strings are words or "no" if one or both are nonwords. There are four types of word pairs. Type 1 word pairs rhyme and are spelled alike except for the first letter (e.g., BRIBE-TRIBE). Type 2 word pairs are neither orthographically nor phonologically similar; they are re-pairings of words of the first type and serve as control pairs for them. Type 3 word pairs consist of words that are spelled alike except for the first letter, but do not rhyme (e.g., FLOWN-CLOWN). Type 4 word pairs consist of control words for these nonrhyming pairs.

This research was supported by Grant NS-18010 from the National Institute of Neurological and Communicative Disorders and Stroke and by Grant HD-01994 from the National Institute of Child Health and Human Development. We are grateful to individuals at Gallaudet College who made it possible for us to conduct the research. In particular we thank Horace Reynolds, Donald Moores, and Pat Cox for their cooperation. We also thank John Richards, Ignatius Mattingly, Rena Krakow, Alvin Liberman, Carol Padden, and Nancy McGarr for their valuable discussions regarding this research, and Nancy Fishbein, Debbie Kuglitsch, and Beth Schwenzfeier for their help in testing subjects. Requests for reprints should be sent to Vicki L. Hanson, Haskins Laboratories, 270 Crown Street, New Haven, CT 06511.
Meyer et al. (1974) argued that if word reading were done on a completely visual basis, the following equation should hold for response times:

$$
\text { Type } 2-\text { Type } 1 \text { = Type } 4-\text { Type } 3 .
$$

If, however, there were a phonological influence, then:

$$
\text { Type } 2 \text { - Type } 1 \neq \text { Type } 4 \text { - Type } 3 \text {. }
$$

The inequality was upheld in their study. Meyer et al. found a small facilitation effect for rhyming words (Type 1) relative to control items of Type 2 . They found a large interference effect for nonrhyming, orthographically similar pairs (Type 3) relative to control items of Type 4 . Because the rhyming and nonrhyming test pairs were equally similar orthographically, the differential outcome on the rhyming and nonrhyming pairs could be ascribed unambiguously to the differences in the phonological relationship between members of the two pair types.

Research subsequent to that of Meyer et al. (1974) has revealed that this pattern of facilitation and interference is dependent on task variables (Evett \& Humphreys, 1981; Shulman, Hornak, \& Sanders, 1978). For example, the pattern has been found to be related to the nonword distractors used in the task: When the nonwords are pronounceable nonwords (i.e., "pseudowords"), the pattern obtained by Meyer et al. is apparent, but when the nonwords are unpronounceable, there is facilitation for orthographically similar word pairs, whether rhyming or nonrhyming (Shulman et al., 1978). These latter findings 
have been used to argue against the notion of an obligatory phonological mediation in lexical access. However, the interpretation that the response time (RT) difference obtained with Meyer et al.'s procedure is caused by the discrepant phonological representations of the nonrhyming pairs of words remains unquestioned.

Our interest in the procedure of Meyer et al. (1974) derives from the information it may provide about word reading by deaf individuals. We ask here whether skilled deaf readers are able to access phonological information about a word under conditions in which skilled hearing readers do so. Therefore, any bias that the procedure may introduce toward accessing phonological information will be to our advantage.

There are at least two ways in which a prelingually, profoundly deaf reader might acquire information about the phonological forms of words. First, the alphabetic orthography itself provides phonological information. According to some theorists (Chomsky \& Halle, 1968; Gleitman \& Rozin, 1977; see also Crowder, 1982), the English orthography maps onto the phonological representations of words most directly at the level of the "systematic phoneme," which, putatively, is the level of phonological representation specified in the lexical entries of mature users of the language (but see Linell, 1979; Steinberg, 1973). Although deaf readers may be able to acquire information about the systematic phonological forms of words from the orthography, any information that the orthography may thus provide will not distinguish the rhyming from the nonrhyming orthographically similar word pairs in Meyer et al.'s (1974) paradigm. For example, there is nothing in the written forms of SAVE and WAVE, on the one hand, and HAVE and CAVE, on the other, that could reveal to a reader otherwise ignorant of the phonological forms of these words that the first pair of words is rhyming and the second pair nonrhyming. A second way in which a deaf reader might acquire information about the phonological forms of words is by learning to speak and/or lip-read the language. This would enable acquisition of a phonetic or classical phonemic representation.

In Experiments 1 and 2, we used Meyer et al.'s (1974) task to ask whether deaf readers access phonological information in a form that leads to facilitation when orthographically similar words rhyme and to interference when they do not. The deaf subjects in our study were college students, and thus, presumably, represented relatively successful deaf readers. To provide baseline data for interpreting the performance of the deaf subjects, a group of hearing college students was also tested.

\section{EXPERIMENT 1}

\section{Method}

Stimuli and Design. The word/word pairs were those used by Meyer et al. (1974; see that article for a more complete discussion of the selection procedures for these pairs). These pairs were of four types. Type 1 (rhyming) word pairs were orthographically and phonologically similar (e.g., MARK-DARK, LOAD-TOAD). Type 2 pairs were control pairs that were both orthographically and phonologically dissimilar. These control pairs were constructed by interchanging the first and second members of the Type 1 pairs (e.g., MARK-TOAD, LOAD-DARK). Type 3 (nonrhyming) pairs were orthographically similar although phonologically dissimilar (e.g., GONE-BONE, PAID-SAID). Type 4 pairs were control pairs for the Type 3 pairs. These Type 4 pairs were both orthographically and phonologically dissimilar and were constructed by interchanging the two members of the Type 3 pairs (e.g., GONE-SAID, PAID-BONE). There were 48 pairs of each of the four types.

In addition to these 192 word/word pairs, 192 word/nonword pairs were constructed by pairing each word of the Type 1 and Type 3 word/word pairs with a pseudoword (pronounceable nonword). The pseudowords were formed by replacing the first letter of each word with a letter that made the string a pseudoword. Thus, as with the word/word pairs, half of the word/nonword pairs were orthographically similar (e.g., MARK-WARK, NAID-PAID), and half were orthographically dissimilar (e.g., ROWN-TOAD, PAID-TOST).

Using these word/word pairs and word/nonword pairs, two stimulus sets of 192 pairs were constructed. Each set contained half of each type of word/word pair and half of the word/nonword pairs. The two sets were constructed so that the words appearing in the Type 1 pairs in one set appeared in the Type 2 pairs of the other set. Similarly, the words that appeared in the Type 3 pairs in one set appeared in the Type 4 pairs of the other set. Thus, no word appeared twice in a word/word pair within either set. The word/nonword pairs of each set contained one member from each of the word/word pairs in the set. For half of the word/nonword pairs, the word appeared first (on top); for the other half, the nonword appeared first. For each stimulus set, a random order of pair presentations was generated, and this list was divided into six blocks of 32 trials each.

Two practice blocks of 32 trials each were generated. The stimulus pairs in these practice blocks were constructed in a manner consistent with the experimental blocks.

Procedure. The start of each trial was signaled by a $250-\mathrm{msec}$ fixation point $(\mathrm{a}+)$ presented in the center of a CRT display. Following this, there was a 250 -msec blank interval prior to stimulus presentation. The two letter strings for a trial were then presented, the first string centered one line above where the fixation cross had appeared and the second string centered one line below that point. The strings remained in view either until the subject pressed a response key or until $5 \mathrm{sec}$ had elapsed.

Feedback was given on each trial. The feedback consisted of the subject's RT (in milliseconds) for that trial, which, if the subject had erred, was preceded by a minus sign. If the subject had failed to respond within the 5-sec time limit, the words TOO SLOW appeared as feedback. The feedback, displayed for $250 \mathrm{msec}$, was centered six lines below the fixation cross. There was approximately a 2.5-sec interval before the start of the next trial.

The subjects were instructed that on each trial they would be presented with two letter strings and that their task was to decide, as quickly and as accurately as possible, whether or not both letter strings were English words. The instructions were written, and the experimenter answered any questions that the subjects had about either the task or the feedback. For the deaf subjects, the experimenter was a recent deaf graduate of Gallaudet College who communicated with the subjects by signing. For the hearing subjects, the experimenter was a hearing person.

The subjects were shown the two response keys, one labeled YES and the other labeled NO. If both letter strings on a trial were English words, the subjects were to press the YES key; if they were not both English words, the subjects were to press the NO key. The subjects were instructed to keep their index fingers resting one on each key to achieve fastest RTs.

All subjects were presented with the two practice blocks, followed by testing with one of the two experimental stimulus sets. In addition, the assignment of the YES/NO keys for the two hands was counterbalanced across subject group and stimulus set.

Following this lexical decision task, the subjects were presented 
with a rhyme judgment task to determine whether deaf readers could distinguish rhyming from nonrhyming pairs of words. The rhyme task was a paper-and-pencil test in which the subjects were required to indicate whether or not the two words of each pair rhymed. Word pairs of Types 1-4 were typed (in lowercase letters) and were followed by a blank line. The written instructions informed the subjects that they were to write "YES" on the blank line if the two words of the pair rhymed and to write "NO" on the line if the two words did not. Two forms of the test were constructed. One form used the word/word pairs from Set 1 and their order of presentation from the lexical decision task; the other form used the word/word pairs of Set 2 in their previous order of presentation. The subjects received the form corresponding to the stimulus set they had received in the lexical decision task.

Deaf subjects. Deaf participants were 16 students from Gallaudet College, a liberal arts college specifically for deaf students. Measures of hearing loss and speech intelligibility were obtained from records at the college. As criteria for inclusion in the experiment, deaf subjects had to be prelingually deaf and have a profound hearing loss. Three of the participants failed to meet these criteria due to postlingual deafness (age 3 years or older) and were dropped from the study. In addition, the data of 1 deaf subject were excluded because of a mean error rate more than 2.5 standard deviations greater than that of the group average. This resulted in 12 deaf stubjects; 11 were congentially deaf, and the other was adventitiously deaf ened before the age of 2 years. Six of these subjects had deaf parents. All had a hearing loss of $90 \mathrm{~dB}$ or greater, better ear average. Half of the subjects were tested with one stimulus set, and the other half with the second set.

The speech intelligibility ratings of the deaf subjects were based on a scale of 1 to 5 , in which 1 was readily intelligible speech and 5 was unintelligible speech. Of the 12 deaf subjects in this experiment, 2 had speech that was rated 3 , meaning that the general public had some difficulty in understanding the speech initially, but could understand it after repeated exposure; 4 of the subjects had speech that was rated 4 , meaning that the speech was very difficult for the public to understand; and 6 of the subjects had speech that was rated 5 , meaning that it could not be understood.

The reading level of the deaf subjects was assessed by means of the comprehension subtest of the Gates-MacGinitie Reading Tests (1969, Survey F, Form 2), which was administered following the rhyme judgment task. Survey $F$ of the test is designed for hearing students in Grades 10 through 12. On this comprehension test, a percentile score was determined for each subject based on Grade Level 10.1. The percentiles ranged from 97 to $7(N=12$, median $=22.5$ ).

Hearing subjects. Hearing subjects were 16 students from Yale University who reported no history of hearing impairment. Eight of these subjects were tested with each experimental set.

\section{Results and Discussion}

For analysis purposes, RTs in the lexical decision task were stabilized by eliminating RTs in each condition that differed from the cell mean by more than two standard deviations. Table 1 provides the mean correct RTs (in milliseconds) and mean percentages of errors for each group and condition.

A difference score was obtained for each subject for phonological similarity (Type 2 minus Type 1) and phonological dissimilarity (Type 4 minus Type 3 ). Table 1 also provides the mean difference scores for the two subject groups. Table 1 shows that the hearing subjects exhibited the response pattern found by Meyer et al. (1974), namely, a small facilitation effect on rhyming
Table 1

Mean Response Times (RTs) (in Milliseconds) and Mean Percentages of Errors (PEs) in the Lexical Decision Task of Experiment 1

\begin{tabular}{lrrrrr}
\hline & \multicolumn{2}{c}{ Hearing } & & \multicolumn{2}{c}{ Deaf } \\
\cline { 2 - 2 } \cline { 5 - 6 } & RT & PE & & RT & PE \\
\hline Word/Word Pairs & & & & \\
Phonologically Similar & 775 & 7.5 & 602 & 13.9 \\
Control & 800 & 4.9 & & 657 & 11.9 \\
$\quad$ Difference Score & 25 & -2.6 & & 55 & -2.0 \\
Phonologically Dissimilar & 845 & 12.7 & 631 & 9.8 \\
Control & 793 & 6.1 & 633 & 11.3 \\
$\quad$ Difference Score & -52 & -6.6 & & 2 & 1.5 \\
Pseudoword/Word Pairs & 931 & 19.6 & 732 & 40.9 \\
\hline
\end{tabular}

Note-A positive number for the difference score indicates facilitation, and a negative number indicates interference.

word pairs and a larger interference effect on nonrhyming pairs. The deaf subjects also responded differentially to the rhyming and nonrhyming pairs, but exhibited a somewhat different response pattern. These subjects showed relatively large facilitation on rhyming words, but neither facilitation nor interference on nonrhyming words.

Using the difference scores, an analysis of variance was performed on the within-subjects factor of phonological relation (similar, dissimilar) and the between-subjects factors of group (deaf, hearing) and stimulus set (Set 1, Set 2). Stimuli were treated as a fixed effect because of the constraints imposed upon stimulus selection in this experiment and in Experiments 2 and 3 (see also Evett \& Humphreys, 1981; Shulman et al., 1978). The factors of interest here are phonological relation and any interaction that may involve subject group.

The analysis of RTs yielded a significant main effect of phonological relation $[F(1,24)=26.17, M S e=$ $2,169.65, p<.001]$, with difference scores in the phonologically similar condition tending to be positive (reflecting facilitation for rhyming word pairs) and difference scores for the phonologically dissimilar condition tending to be negative (reflecting interference). This main effect did not interact with either subject group or stimulus set (both $F$ s $<1$ ). Thus, there was no significant difference in the magnitude of the effect of phonological relation for the hearing and deaf subjects. The higher order interaction involving these variables also was not significant $(F<1)$. This pattern of RTs is inconsistent with the hypothesis that graphemic information alone is utilized in this task by either hearing or deaf subjects. A main effect of group $[F(1,24)=5.24, M S \mathrm{e}=4,675.98, p<.05]$ reflected the fact that for the hearing subjects the mean of the difference scores was negative (reflecting a large interference effect and a smaller facilitation effect), whereas for the deaf subjects the mean of the difference scores was positive (reflecting only a facilitation effect).

An analysis of variance on the difference scores for the error data revealed no significant main effects or interactions (all $p s>.05$ ).

In the rhyme judgment task, the deaf subjects made many errors, particularly on the orthographically similar but phonologically dissimilar (Type 3 ) word pairs. A simi- 
Table 2

Mean Percentages of Errors for Deaf and Hearing Subjects in the Rhyme Judgment Task

\begin{tabular}{lcccc}
\hline & Type 1 & Type 2 & Type 3 & Type 4 \\
\hline Deaf & 28.1 & 5.6 & 70.8 & 3.5 \\
Hearing & 2.3 & .8 & 11.2 & .8 \\
\hline
\end{tabular}

lar error pattern was obtained for the hearing subjects, although their error rate was much lower. Table 2 shows the mean percentages of errors for each word type for hearing and deaf subjects. This pattern of responding suggests that the subjects' responses in this task were influenced by the orthographic similarity of the stimulus pairs. In fact, 1 deaf subject exemplified this strategy perfectly, by not making any erors on the Type 1 (orthographically and phonologically similar) word pairs on the rhyme judgment task but making an error on each of the 24 Type 3 pairs. One hearing subject showed much the same pattern by not making any errors on the Type 1 pairs and making errors on 17 of the 24 Type 3 pairs in this task.

For the deaf subjects, correlations were computed between their speech intelligibility rating, reading achievement, accuracy on the rhyme judgment task, and RTs on the lexical decision task. The only correlation to reach significance was the correlation between speech intelligibility and errors on Type 3 word pairs on the rhyme judgment task $[r(10)=-.81, p<.01$, two-tailed], which indicated that the more intelligible the speech, the greater the accuracy on these pairs. Other correlations with speech intelligibility, although not significant (all $p s>.10$, twotailed), were in the expected direction: The better the rated speech intelligibility, the greater the overall accuracy on the rhyme judgment task $(r=-.32)$ and the larger the $\mathrm{RT}$ effect of phonological relation in the lexical decision task $(r=-.47)$.

\section{EXPERIMENT 2}

Experiment 2 was similar to Experiment 1, except for differences in the stimulus set, instructions to the subjects, and the form of rhyme judgment task.

We changed the stimulus set to determine whether the findings of Experiment 1 would be replicated, an approach Wike and Church (1976) proposed for showing generalization over stimuli. In our new stimulus set, we attempted to control for possible differences in the size of the orthographic neighborhoods of rhyming and nonrhyming words by selecting pairs of rhyming and nonrhyming words from a common neighborhood. For example, one rhyming pair of words in Experiment 2 was DONE-NONE; the corresponding nonrhyming pair was BONE-GONE.

The change in instructions was motivated by the high error rate among the deaf subjects in Experiment 1; in Experiment 2, we requested that the subjects try to maintain a level of accuracy of $90 \%$ or better.
The change in the rhyme judgment task was designed to force deaf subjects to try to make their judgments on the basis of phonological information rather than orthographic similarity. As noted in Experiment 1, the deaf subjects and one hearing subject identified most of the Type 3 words as rhyming. We thought that this manner of responding might have been promoted by the fact that just one fourth (rather than one half) of the word pairs in the test rhymed. In Experiment 2, therefore, only pairs of Types 1 and 3 were included in the rhyming test. In addition, words were presented in matched pairs (e.g., DONE-NONE was presented with BONE-GONE), and the subjects had to select which of the two matched word pairs rhymed.

\section{Method}

Stimuli and Design. The word/word pairs were chosen so that for each rhyming pair (Type 1) there was a nonrhyming pair (Type 3) that was orthographically similar (e.g., SAVE-WAVE and HAVE-CAVE; DONE-NONE and BONE-GONE). There were 32 such matched pairs. These stimuli are given in the Appendix.

In all other respects, the design of this experiment followed that of Experiment 1. Rhyme controls (Type 2 words) were generated by repairing the Type 1 words. Nonrhyme controls (Type 4 words) were generated by repairing the Type 3 words. Pseudowords were formed by replacing the first letter of each word with a letter that made the string a pronounceable nonword. In total, there were 128 word/word pairs and 128 pairs in which one of the items was a pseudoword.

Two stimulus sets were constructed. Assignment of pairs to a list was made as in Experiment 1, with the one additional constraint that the matched orthographically similar pairs never both occur in the same list. Half of the Type 1 and Type 3 word pairs occurred in one set, and the remaining word pairs in the other set. For each stimulus set, a random order of pair presentations was generated and presented as four blocks of 32 trials each.

Two practice blocks of 32 trials each were constructed in a manner consistent with list construction in the experimental blocks.

Procedure. The lexical decision task procedure was identical to that in Experiment 1, except that instructions to the subjects stressed accuracy. The subjects in both groups were told to try to be at least $90 \%$ accurate. All subjects pressed the YES response key with the right hand and the NO response key with the left hand.

Following the lexical decision task, the subjects were asked to complete a rhyme judgment task. This rhyming task was a paperand-pencil test that consisted of 32 trials using pairs of words from the lexical decision task. On each trial, two word pairs were presented, one pair to the right of the other. The two words of each pair were orthographically similar, but words in one pair on each trial rhymed (a Type 1 pair) and the words in the other did not (a Type 3 pair). The two pairs on each trial were always the ones matched for orthographic similarity. Thus, for example, the subjects had to indicate whether the pair SAVE-WAVE or the pair HAVE-CAVE rhymed. A short blank line preceded each pair. The subjects were told that on each trial one of the two pairs rhymed. They were to indicate which of the two pairs rhymed by making a check on the line in front of the rhyming pair. For each subject, one of the two pairs on each trial had been tested in the lexical decision task; half of these previously seen stimuli were rhymes (Type 1 pairs), and half were nonrhymes (Type 3 pairs).

Deaf subjects. The deaf subjects were 16 students from Gallaudet College. Two of the deaf subjects were eliminated from the study because of postlingual deafness (age 3 years) and 1 because of a 
Table 3

Mean RTs (in Milliseconds) and Mean Percentages of Errors (PEs) in the Lexical Decision Task of Experiment 2

\begin{tabular}{lrrrrr} 
& \multicolumn{2}{c}{ Hearing } & & \multicolumn{2}{c}{ Deaf } \\
\cline { 6 - 6 } & RT & PE & & RT & PE \\
\hline Word/Word Pairs & & & & \\
$\quad$ Phonologically Similar & 778 & 7.5 & & 972 & 8.7 \\
Control & 804 & 11.6 & 1026 & 10.1 \\
$\quad$ Difference Score & 26 & 4.1 & 54 & 1.4 \\
Phonologically Dissimilar & 848 & 15.2 & 1003 & 9.9 \\
Control & 801 & 10.3 & & 986 & 9.7 \\
$\quad$ Difference Score & -47 & -4.9 & -17 & -.2 \\
Pseudoword/Word Pairs & 804 & 14.8 & 1078 & 16.0 \\
\hline
\end{tabular}

Note-A positive number for the difference score indicates facilitation, and a negative number indicates interference.

reported hearing loss less than the criterion of $85 \mathrm{~dB}$. The data of 1 deaf subject were eliminated because of excessive error rate (more than 2.5 standard deviations greater errors than the mean for the deaf subject group). Eleven of the remaining 12 subjects were congenitally deaf, and the other was adventitiously deafened before the age of 1 year. Four of these subjects had deaf parents. Five were tested on Set 1 and 7 on Set 2 .

Speech intelligibility ratings were available for 11 of the 12 subjects. One of these subjects had speech that was rated 2, 3 had speech that was rated 3, 4 had speech that was rated 4, and 3 had speech that was rated 5 .

After the lexical decision and rhyme judgment tasks, we assessed the reading level of the deaf subjects by means of the comprehension subtest of the Gates-MacGinitie Reading Tests (1978, Level F, Form 2), designed for hearing students of Grades 10-12. The percentile scores for the subjects ranged from 97 to $10(N=12$, median $=48$ ).

Hearing subjects. The hearing subjects were 14 students from Yale University. Seven subjects were tested with each stimulus set.

\section{Results and Discussion}

Consistent with the analyses in Experiment 1, RTs that differed by more than two standard deviations from a subject's mean in each cell were discarded. Table 3 shows the means (in milliseconds) for the correct RTs and the mean percentages of errors for the two subject groups in each condition. Also shown are the difference scores for phonological similarity and dissimilarity. As can be seen from Table 3, the performance of the hearing subjects in Experiment 2 was remarkably similar to that of the hearing subjects in Experiment 1 . The deaf subjects in Experiment 2 were slower and more accurate than those in Experiment 1, presumably because of our change in instructions to emphasize accuracy. Despite this change in position along the speed-accuracy continuum, the deaf subjects showed a pattern similar to that exhibited by the deaf subjects in Experiment 1, namely, a large facilitation on the rhyming pairs but little interference on the nonrhyming pairs.

The analysis of the RT difference scores indicated a main effect of phonological relation $[F(1,22)=6.20, M S \mathrm{e}$ $=9,742.00, p<.05]$ that did not significantly interact with group $(F<1)$. No other main effects or interactions were significant (all $p s>.25$ ). The main effect of phonological similarity reflected the fact that for rhym- ing pairs there was RT facilitation, whereas for nonrhyming pairs there was RT interference. This result indicated that both hearing and deaf subjects were influenced by the phonological similarity of the word pairs. The magnitude of the phonological similarity effect was not significantly different for the deaf and hearing subjects.

The analysis of the error data also indicated a main effect of phonological relation $[F(1,22)=7.09, M S e=$ $38.98, p<.05]$ and interactions of this variable with stimulus set $[F(1,22)=12.63, p<.01]$ and subject group $[F(1,22)=6.24, p<.05]$. The main effect resulted from fewer errors on the rhyming items than on the control and from more errors on the nonrhyming items than on the control. The interaction with set reflected a larger influence of phonological relationship for one stimulus set than for the other. The interaction with subject group reflected a larger influence of phonological relationship for hearing subjects than for deaf subjects.

The lack of an interference effect among the deaf subjects in Experiment 1, and the relatively small interference effect among these subjects in Experiment 2, may have one of two origins. First, it may be that the deaf subjects individually, as well as collectively, showed no interference. Alternatively, some deaf subjects may have shown interference, whereas others, failing to distinguish rhyming words from orthographically similar nonrhyming words, showed facilitation on both sets of words. To distinguish between these two possibilities, we looked at individual performances in the rhyming and nonrhyming conditions in Experiments 1 and 2. As a comparison, we also looked at individual hearing subjects.

The results of this classification are more in line with the second alternative. As shown in Table 4, the individual responses revealed that for both hearing and deaf subjects, slightly fewer than half of the subjects showed facilitation on the phonologically similar word pairs and interference on the phonologically dissimilar word pairs. The magnitudes of these facilitation and interference effects, as shown in Figure 1, were similar for the hearing and the deaf subjects (with the possible exception of the deaf subjects in Experiment 2, who actually showed a larger interference effect than the hearing subjects). Further in-

Table 4

Results of the Analysis of Individual Subjects' Data in Experiments 1 and 2

\begin{tabular}{lrrrrr}
\hline & \multicolumn{3}{c}{ Phonologically Dissimilar } \\
\cline { 5 - 6 } & \multicolumn{2}{c}{ Interference } & & \multicolumn{2}{c}{ Facilitation } \\
\cline { 6 - 6 } \cline { 5 - 6 } Phonologically Similar & Exp. 1 & Exp. 2 & & Exp. 1 & Exp. 2 \\
\hline Facilitation & & & & & \\
$\quad$ Hearing & 50 & 43 & & 25 & 14 \\
$\quad$ Deaf & 42 & 33 & & 50 & 50 \\
Interference & & & & \\
$\quad$ Hearing & 25 & 36 & & 7 \\
$\quad$ Deaf & 8 & 0 & & 0 & 17 \\
\hline
\end{tabular}

Note-Table 4 shows the mean percentages of hearing and deaf subjects whose RTs revealed facilitation or interference as a function of whether the word pairs were rhyming (phonologically similar) or nonrhyming (phonologically dissimilar). 


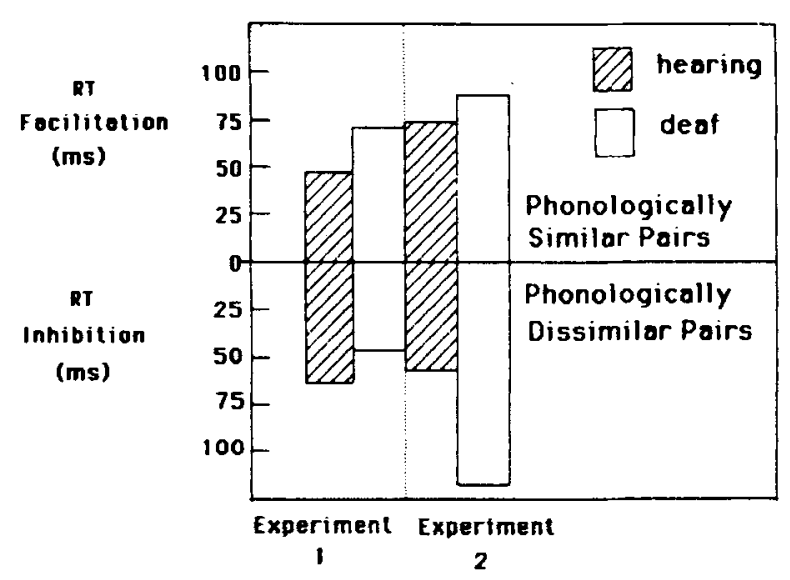

Figure 1. Mean response time (RT) difference scores (in milliseconds) for the deaf and hearing subjects in Experiments 1 and 2 who showed both facilitation on rhyming (Type 1) word pairs and interference on nonrhyming (Type 3 ) word pairs.

spection of the individual responses revealed that the differences in pattern in the group data resulted from the fact that more deaf than hearing subjects exhibited facilitation on both of these word types, whereas more hearing than deaf subjects exhibited interference on both the phonologically similar and dissimilar pairs.

The results of the rhyme judgment task indicated that the hearing subjects more accurately discriminated between the rhyming and nonrhyming orthographically similar pairs than did the deaf subjects; the mean percentages of correct responses were $99.6 \%$ and $64.1 \%$ for hearing and deaf subjects, respectively. Despite the fact that the deaf subjects thus made a considerable number of errors, their performance was significantly better than chance in this two-choice task $[t(11)=4.05, p<.002]$.

For the deaf subjects, further analyses yielded no significant correlations between individual subject characteristics (speech intelligibility and reading achievement) and accuracy on the rhyme judgment task or RT on the lexical decision task. The correlation between speech intelligibility and accuracy on the rhyme task was in the same direction as that in Experiment $1[r(10)=-.56$, $.05<p<.10$, two-tailed]. The correlation between speech intelligibility and phonological relation in the lexical decision task was in the same direction as that in Experiment 1 , although small $(r=-.13)$.

\section{EXPERIMENT 3}

In Experiments 1 and 2, both the hearing and the deaf subjects responded differentially to rhyming and nonrhyming orthographically similar word pairs in the lexical decision task. This pattern of differential facilitation as a function of phonological similarity shown by both deaf and hearing subjects is consistent with the notion that subjects in both of these groups were accessing phonological information. ${ }^{1}$ This outcome for hearing subjects is not remarkable, but it is surprising that prelingually, pro- foundly deaf subjects showed evidence of access to phonological information.

To substantiate our conclusion that the response pattern of deaf subjects indeed reflects access to phonological information, we next used a manipulation that does not change the word/word pairs but has been reported to change hearing subjects' pattern of performance in the paradigm of Meyer et al. (1974). This manipulation, performed by Shulman et al. (1978), uses consonant strings as nonword distractors. With hearing subjects, Shulman et al. found that this manipulation facilitated responding on orthographically similar nonrhyming (Type 3 ) as well as on rhyming (Type 1) word pairs. The result was taken as evidence that the use of phonological information was reduced. The finding of semantic priming by Shulman et al. with orthographically and phonologically irregular nonwords indicated that subjects were accessing the lexicon in their task, not simply truncating the decision process after determining the regularity of the letter string.

If the effects obtained for the deaf subjects in Experiments 1 and 2 could possibly be attributed to unidentified nonphonological factors (e.g., visual similarity or sign similarity differences in the rhyming and nonrhyming word pairs), then changing only the nonword distractors in Experiment 3 was not expected to influence their pattern of responding; that is, they were expected to continue to show facilitation on the rhyming but not on the nonrhyming pairs. If the pattern of results obtained for the deaf subjects in Experiments 1 and 2 was attributable to the phonological relationships between the two words of a pair, then when the distractor items were orthographically and phonologically irregular the deaf subjects, like the hearing subjects in Shulman et al.'s (1978) study would be expected to show facilitation on both the rhyming (Type 1) and the nonrhyming (Type 3) word pairs.

In Experiment 3, therefore, we used the word/word pairs of Experiment 1, but altered the pseudowords so that they were orthographically and phonologically irregular strings. Thus, the only difference between the stimuli of Experiments 1 and 3 was in the distractor items. Any difference in responding in the two experiments could therefore be attributed to this change.

\section{Method}

Stimuli and Design. The two stimulus sets (and the two practice blocks) of Experiment 1 were used, and the items were presented in the same order as in Experiment 1. The word/word pairs were identical to those of Experiment 1. The pseudowords of experiment 1 were changed to consonant strings by replacing each vowel in a pseudoword with a consonant.

Procedure. The procedure in the lexical decision task was identical to that of Experiment 2, with the exception that no specific mention of accuracy was made.

Following the lexical decision task, the subjects were again given a rhyme judgment task. This task was similar to that of Experiment 2 in that on each trial the subjects had to indicate which of two orthographically similar pairs rhymed. All of the rhyming (Type 1) and nonrhyming (Type 3) pairs from the two stimulus sets were used, resulting in 48 pairs. On each trial, both pairs were from the same set. Two forms of the test were constructed; the same word 
pairs were used, but the rhyming and nonrhyming pairs were repaired. These two forms of the test were given to different subjects.

Deaf subjects. The deaf subjects were 15 students from Gallaudet College. As in Experiments 1 and 2, the criteria for inclusion in the study were that subjects be prelingually, profoundly deaf. Two subjects were excluded because of postlingual deafness (age 3 years or older), and 1 was eliminated because of a hearing loss less than the criterion of $85 \mathrm{~dB}$. Twelve experimental subjects remained. Eleven of these subjects were congenitally deaf, and the other had been deafened before the age of 2 years. Two of these subjects had deaf parents. Five subjects were tested in experimental Set 1 , and 7 in Set 2 . Due to experimenter error, 3 of the 12 subjects were not given the rhyme judgment task.

The speech intelligibility ratings were available from Gallaudet College for all but 2 of the subjects. The ratings for the remaining subjects were as follows: One subject had a rating of 2,2 subjects had a rating of 3,5 subjects had a rating of 4 , and 2 subjects had a rating of 5 .

Due to experimenter error, reading tests were given to only 9 of the subjects in this experiment. Of those 9, 4 were given the comprehension test used in Experiment 1 (Gates-MacGinitie Reading Tests, 1969, Survey F, Form 2), and 5 were given the comprehension test of the more recent version of the test used in Experiment 2 (Gates-MacGinitie Reading Tests, 1978, Level F, Form 2). The percentile scores for these subjects in relation to Grade 10.1 ranged from 79 to $9(N=9$, median $=49)$.

Hearing subjects. The hearing subjects were 15 students from the University of Connecticut. Seven were tested with stimulus Set 1, and 8 with Set 2 .

\section{Results and Discussion}

As in Experiments 1 and 2, RTs that differed by more than two standard deviations from a subject's mean in each cell were eliminated from analysis. Table 5 shows the means (in milliseconds) for the correct RTs and the mean percentages of errors for the two subject groups in each condition. Table 5 also shows the difference scores for phonological similarity and dissimilarity. The difference scores for each of the two subject groups indicated facilitation on both the phonologically similar (Type 1) and phonologically dissimilar (Type 3) words. As Tables 1, 3 , and 5 show, changing the pseudowords to consonant strings resulted in an increased facilitation on rhyming pairs for both hearing and deaf subjects. Moreover, it resulted in facilitation of the nonrhyming, but orthographically similar, pairs as well.

Table 5

Mean RTs (in Milliseconds) and Mean Percentages of Errors (PEs) in the Lexical Decision Task of Experiment 3

\begin{tabular}{lrrrrr}
\hline & \multicolumn{3}{c}{ Hearing } & & \multicolumn{2}{c}{ Deaf } \\
\cline { 2 - 3 } \cline { 6 - 7 } & RT & PE & & RT & PE \\
\hline Word/Word Pairs & & & & \\
Phonologically Similar & 592 & 2.1 & & 542 & 5.1 \\
Control & 707 & 6.1 & & 645 & 12.6 \\
$\quad$ Difference Score & 115 & 4.0 & & 103 & 7.5 \\
Phonologically Dissimilar & 605 & 2.4 & & 537 & 1.8 \\
Control & 681 & 5.2 & & 603 & 11.1 \\
$\quad$ Difference Score & 76 & 2.8 & & 66 & 9.3 \\
Nonword/Word Pairs & 633 & 8.3 & & 558 & 14.8 \\
\hline
\end{tabular}

Note-A positive number for the difference score indicates facilitation.
The RT difference scores were entered into an analysis of variance on the factors of phonological relation, stimulus set, and subject group. The analysis indicated a main effect of phonological relation $[F(1,23)=6.28$, $M S e=2,955.98, p<.02]$. No other main effects or interactions were significant (all $p s>.50$ ). The main effect of phonological relation indicated that although there was facilitation for both the rhyming and nonrhyming words, the facilitation was greater for the rhyming pairs.

The analysis of difference scores for errors indicated no significant effects (all $p s>.25$ ).

The pattern of RTs in Experiment 3 differed from the patterns of Experiments 1 and 2 in that orthographic similarity facilitated responding, regardless of whether the words of a pair were phonologically similar or dissimilar. This was Shulman et al.'s (1978) finding, which they interpreted as evidence that access to phonological information is eliminated with consonant strings as nonwords. One aspect of our outcome leads us to treat this interpretation with caution. In Experiment 3, the effect of phonological similarity, for both hearing and deaf subjects, was still significant, albeit numerically somewhat smaller than in Experiments 1 and 2. Evidently, Shulman et al.'s procedures did not eliminate the influence of phonological information. Shulman et al. obtained the same pattern. In their experiments, too, there was greater facilitation for the orthographically similar rhyming than nonrhyming pairs when irregular nonwords were used. Although the difference was not statistically significant in their study, the differences they obtained with irregular nonwords were consistent in direction and magnitude with the differences obtained here; the facilitation was greater for the rhyming pairs by $37 \mathrm{msec}$ in their Experiment 1, by $31 \mathrm{msec}$ in their Experiment 2, and by $24 \mathrm{msec}$ in their Experiment 3. In the present experiment, the facilitation was greater for the rhyming pairs by an average of $38 \mathrm{msec}(39 \mathrm{msec}$ for the hearing subjects and $37 \mathrm{msec}$ for the deaf subjects).

Rather than eliminating access to phonological information, the inclusion of the consonant strings as nonwords appears to have increased reliance on orthographic information in responding. This increased reliance on orthographic information can be seen as a criterion shift in Experiment 3, a shift leading to fast rejection and somewhat quick acceptances. Comparison of RTs in Tables 1, 3, and 4 shows faster RTs in Experiment 3, particularly on nonwords. Shulman et al. (1978) also obtained this faster responding with orthographically and phonologically illegal nonwords.

The results of the rhyme judgment task were similar to those in Experiment 2. The deaf subjects were considerably less accurate than the hearing subjects, but again were better than chance. The mean percentages of correct responses were $99.4 \%$ and $60.2 \%$ for hearing and deaf subjects, respectively. Despite the fact that the deaf subjects made a considerable number of errors, their performance was significantly better than chance in this twochoice task $[t(8)=3.21, p=.02]$. 
For the deaf subjects, correlations were small and nonsignificant between individual subject characteristics (speech intelligibility and reading achievement) and accuracy on the rhyme task or RT on the lexical decision task. The correlation between speech intelligibility and accuracy on the rhyme task was in the same direction as the correlations in Experiments 1 and $2(r=-.19)$. The correlation between speech intelligibility and phonological relation in the lexical decision task was essentially zero $(r=-.03)$. The failure consistently to obtain significant correlations across the three experiments may be attributable to the restricted range of speech intelligibility scores and the relatively small numbers of subjects in the experiments.

\section{GENERAL DISCUSSION}

The evidence from these studies suggests that deaf readers have access to phonological information in word reading (see Note 1). In the lexical decision tasks of all three experiments, the responses of both hearing and deaf subjects were affected by the phonological relationship between the orthographically similar pairs. This result was obtained using two different sets of word/word pairs (Experiments 1 and 2) and even when consonant strings were used as nonwords (Experiment 3).

The results obtained here argue against the possibility that the deaf subjects' differential responding to rhyming and nonrhyming pairs could have resulted from differences in the visual similarity or the sign similarity of these pairs. The first argument against these interpretations is that Experiments 1 and 3 used the same word/word pairs; only the nonwords differed. This manipulation, although obviously not altering the visual or sign similarity of the word pairs, did alter the deaf (and hearing) subjects' pattern of responding. A second argument against a visual similarity interpretation is that, with the visual similarity of the rhyming and nonrhyming pairs tightly controlled in Experiment 2, the same pattern of results was obtained with two different sets of word/word pairs. A second argument against a sign similarity interpretation is that there is no correspondence between American Sign Language signs and English phonology. There is no reason to expect, therefore, that the rhyming (Type 1) pairs of the experiments should be signed similarly and the nonrhyming (Type 3) pairs should not. Indeed, inspection of the word pairs used in these experiments showed that only one rhyming pair (and no nonrhyming pairs) could be considered to have similar signs. ${ }^{2}$

The major difference in the performance of the two groups was that, overall, the deaf subjects in Experiments 1 and 2 showed more facilitation on the rhyming pairs and less interference on the nonrhyming pairs than did the hearing subjects. Inspection of the individual patterns of performance in Experiments 1 and 2 showed, however, that some deaf subjects did exhibit both facilitation and interference comparable to that of the hearing subjects. The difference between the deaf and hearing subjects in the group data can be accounted for, primarily, by the tendency of some deaf subjects to show facilitation on both the rhyming and nonrhyming pairs and, secondarily, by the tendency of a few hearing subjects to show interference on both types of word pairs. Thus, there were many subjects, in both the hearing and deaf groups, whose patterns of facilitation and interference evidenced the use of phonological information; there were also some subjects in both groups whose response patterns indicated that they failed to distinguish the rhyming from the nonrhyming pairs. There was some suggestion that the pattern of facilitation and interference for the deaf subjects was related to rated speech intelligibility: Those subjects who had the better rated speech showed the larger effects of phonological relation.

An outcome of the present study that requires further consideration is the deaf readers' performance on the rhyme task. In Experiment 1, their response patterns indicated a strong tendency to rely on orthographic similarity in making their rhyme judgments. This finding is consistent with other work on deaf individuals' explicit judgments of rhyme (e.g., Blanton, Nunnally, \& Odom, 1967). However, in the rhyming tasks of Experiments 2 and 3 , in which subjects were forced to make a rhyming judgment without relying on orthographic information, the deaf subjects demonstrated that they could make these judgments with better than chance accuracy.

Two features of the present study are particularly striking. The first is that not only were the deaf subjects accessing phonological information, but that they also were doing so in a speeded task. It might be supposed that deaf readers are confined to accessing phonological information in situations in which they have time to laboriously recover learned pronunciations. In the present study, however, they were found to access phonological information quite rapidly, which suggests that such accessing is a fundamental property of reading.

The second striking feature of this study is that the deaf subjects were not from predominantly oral backgrounds. All had received speech instruction in school, but considered sign to be their primary language. It is noteworthy that in their reading of English they utilized their phonological abilities. In this, the present results converge with evidence from short-term memory studies in which deaf readers, most notably the better ones, were sensitive to phonological similarity manipulations (Conrad, 1979; Hanson, 1982; Hanson, Liberman, \& Shankweiler, 1984; Lichtenstein, in press).

We cannot determine from our research the nature of the deaf readers' phonological representations of words. We can conclude only that their representations of words must include phonological as well as orthographic information. Our findings are compatible with any hypothesized type of phonological representation as long as it captures the phonological similarity of our rhyming pairs and the dissimilarity of the nonrhyming pairs.

The representation could correspond closely to the detailed articulatory form of the word, or it could be more abstract. An articulatory representation would not be incompatible with our findings that phonological informa- 
tion is accessed even by those deaf subjects whose speech is only poorly intelligible. It may well be the case that deaf individuals' ability to use some form of speech-based representation when reading is not well reflected in the intelligibility ratings of their speech. These intelligibility ratings are based on listeners' ability to understand the deaf speakers' utterances, not on the deaf individuals' ability to utilize speech in reading. Further research will be required to discriminate the type of phonological representation used.

In summary, the present study indicates that deaf readers access phonological information. As such, the results run counter to claims that deaf individuals are limited to the use of visual strategies in reading. In interpreting these results, however, it is necessary to bear in mind that the deaf subjects in this study were college students, thus being some of the best educated of deaf individuals. Therefore, these results do not necessarily indicate that the use of phonological information is typical in the reading of deaf individuals. Rather, they indicate that access to this information is possible despite prelingual and profound hearing impairment. Given the impoverished auditory experience of such readers, these results suggest that the use of phonological information need not be tied to the auditory modality.

\section{REFERENCES}

Blanton, R. L., Nunnally, J. C., \& Odom, P. B. (1967). Graphemic, phonetic, and associative factors in the verbal behavior of deaf and hearing subjects. Journal of Speech \& Hearing Research, 10, 225-231.

Chomsky, N., \& Halle, M. (1968). The sound pattern of English. New York: Harper \& Row.

ConRaD, R. (1979). The deaf school child. London: Harper \& Row. CROWDER, R. G. (1982). The psychology of reading: An introduction. New York: Oxford University Press.

Evert, L. J., \& Humphreys, G. W. (1981). The use of abstract graphemic information in lexical access. Quarterly Journal of Experimental Psychology, 33A, 325-350.

Gates-MacGinitie Reading Tests. (1969). New York: Columbia Teachers" College Press.

Gates-MacGinitie Reading Tests (2nd ed.) (1978). Boston: Houghton Mifflin.

Gleitman, L. R., \& Rozin, P. (1977). The structure and acquisition of reading: I. Relations between orthographies and the structure of language. In A. S. Reber \& D. L. Scarborough (Eds.), Toward a psychology of reading. Hillsdale, NJ: Erlbaum.

Hanson, V. L. (1982). Short-term recall by deaf signers of American Sign Language: Implications for order recall. Journal of Experimental Psychology: Learning, Memory, \& Cognition, 8, 572-583.

Hanson, V. L., Liberman, I. Y., \& Shankweiler, D. (1984). Linguistic coding by deaf children in relation to beginning reading success. Joumal of Experimental Child Psychology, 37, 378-393.

Lichtenstein, E. H. (in press). The relationships between reading processes and English skills of deaf college students: Part II. Applied Psycholinguistics.

LINELL, P. (1979). Psychological reality in phonology. Cambridge: Cambridge University Press.

Meyer, D. E., Schvaneveldt, R. W., \& Ruddy, M. G. (1974). Func- tions of graphemic and phonemic codes in visual word-recognition. Memory \& Cognition, 2, 309-321.

Shulman, H. G., Hornak, R., Sanders, E. (1978). The effects of graphemic, phonetic, and semantic relationships on access to lexical structures. Memory \& Cognition, 6, 115-123.

STEINBERG, D. (1973). Phonology, reading and Chomsky's optimal orthography. Journal of Psycholinguistic Research, 2, 239-258.

Wike, E. L., \& Church, J. D. (1976). Comments on Clark's “The language-as-fixed-effect fallacy." Journal of Verbal Learning \& Verbal Behavior, 15, 249-255.

\section{NOTES}

1. We do not mean to imply by this that deaf readers are using only phonological information when they read. We focus on their use of phonological information only because it is so remarkable, given orthographic presentation of items to deaf individuals with poor speech intelligibility.

2. The signs for the rhyming pair TOUGH-ROUGH in Experiment 2 are similarly produced. They are made with similar movement and location, but differ in handshape.

\section{APPENDIX} Word Pairs of Experiment 2

\begin{tabular}{ll}
\hline \multicolumn{1}{c}{ Type 1 } & \multicolumn{1}{c}{ Type 3 } \\
\hline SAVE-WAVE & HAVE-CAVE \\
DONE-NONE & BONE-GONE \\
RUSH-GUSH & HUSH-BUSH \\
GOOD-WOOD & FOOD-HOOD \\
CARD-HARD & WARD-LARD \\
YARN-BARN & EARN-DARN \\
LIGHT-MIGHT & EIGHT-FIGHT \\
TON-WON & CON-SON \\
GULL-LULL & DULL-PULL \\
LORD-FORD & WORD-CORD \\
MATCH-PATCH & CATCH-WATCH \\
KID-BID & AID-RID \\
ROSE-HOSE & NOSE-LOSE \\
NEAR-REAR & DEAR-WEAR \\
HINT-TINT & MINT-PINT \\
MAID-RAID & PAID-SAID \\
SO-NO & GO-DO \\
DOVE-LOVE & MOVE-COVE \\
PUNT-HUNT & AUNT-RUNT \\
TOUGH-ROUGH & COUGH-DOUGH \\
TAR-FAR & BAR-WAR \\
FIVE-DIVE & HIVE-GIVE \\
HOST-POST & LOST-MOST \\
COW-VOW & NOW-LOW \\
RASH-DASH & CASH-WASH \\
CUT-BUT & PUT-NUT \\
HAND-LAND & WAND-SAND \\
TOMB-WOMB & BOMB-COMB \\
FEW-PEW & SEW-NEW \\
BAT-HAT & CAT-OAT \\
DOWN-GOWN & MOWN-TOWN \\
FAST-PAST & EAST-LAST \\
\hline & \\
\hline & \\
&
\end{tabular}

(Manuscript received March 18, 1986; revision accepted for publication September 30, 1986.) 